\title{
El tamaño de las semillas de Vismia glaziovii Ruhl. (Guttiferae) y su relación con la velocidad de germinación y tamaño de la plántula
}

\section{Vismia glaziovii Ruhl. (Guttiferae) seed size and its relationship with the germination speed and seedling size}

\author{
Luis L. Arteaga
}

Estación Biológica Tunquini, Instituto de Ecología, Casilla 10077, Correo Central, La Paz, Bolivia. E-mail Luis L. Arteaga: larteagabohrt@yahoo.com

Presentado: $\quad 04 / 11 / 2005$ Aceptado: $\quad 31 / 01 / 2007$

\section{Resumen}

En el presente trabajo analicé la variabilidad intraespecifica del tamaño de las semillas de Vismia glaziovii Ruhl y su posible efecto sobre la velocidad de germinación y el tamaño de las plántulas. Los resultados muestran que el tamaño de las semillas no varía significativamente entre árboles de las que provenian, pero la variación del tamaño de semilla entre los frutos de cada árbol sí es significativa. También, el tamaño de la semilla no influye sobre la velocidad de germinación ni sobre el tamaño de las plántulas. Sugerimos que la variabilidad en tamaño no sea suficiente como para indicar diferencias en la cantidad de recurso almacenado. Este estudio permitirá contribuir al conocimiento de algunos aspectos de la dinámica de semillas y la regeneración de esta especie.

Palabras clave: Vismia glaziovii, tamaño de semillas, germinación, tamaño de plántulas, bosque montano, Bolivia.

\section{Abstract}

In the present work I analyzed the variability of the seeds size of Vismia glaziovii Ruhl. and its possible effect on germination speed and its seedling size. The results show that the size of the seeds does not vary significantly between trees from which they came; but the variation as large as seed between the fruits of each tree is significant. Also, the size of the seed does not influence the speed of germination nor the seedling size. We suggested that variability in size is not sufficient as indicator of differences in the amount of stored resource. This study will allow contributing to the knowledge of some aspects of the dynamics of seeds and the regeneration of this species.

Keywords: Vismia glaziovii, size seed, germination, size seedling, Bolivia, montane forest.

\section{Introducción}

Las semillas de diversas especies pueden variar en tamaño, forma, estructura del embrión y presencia de tejidos de almacenamiento (Hartmann y Kester, 1982). De estos, el tamaño de la semilla es considerado un factor importante en la ecología de la especie (Leishman et al., 2000); este factor puede influir sobre la velocidad de germinación (Bockus y Shroyer, 1996) y una serie de atributos de la plántula, particularmente el tamaño (Landford y Baker, 1986; Dalling, 2002). Se ha determinado que especies con semillas grandes producen plántulas más vigorosas en el sotobosque, mientras que especies con semillas pequeñas, con rápida germinación, estarían adaptadas a la colonización de nuevos espacios (Snow, 1971).

La variación intraespecifica del tamaño de las semillas ha sido bien estudiada en otras latitudes, sin embargo en las zonas tropicales son escasos estos estudios (Dalling, 2002). Dentro de una misma especie, la variación en el tamaño de las semillas puede deberse a variaciones en el número de semillas producidas por cada fruto o a condiciones ambientales particulares como por ejemplo la humedad del suelo (Dalling, 2002).

Por un lado, Cuya y Lombardi (1991) encontraron que el tamaño de las semillas puede estar inversamente correlacionado con la velocidad de germinación dentro de la misma especie; y por otro lado, varios trabajos han encontrado una correlacion positiva con el tamaño de la plántula, por ejemplo para Virola surinamensis, V. koschnyi, Ludwigia leptocarpa, Desmodium paniculatum, Panicum virgatum, Prunella vulgaris, Quercu mongolica, Prunus virginiana (Howe y Richter, 1982; Dolan, 1984; Wulff,
1986; Winn, 1988;1991; Zhang y Maun, 1991; González, 1993; Moegenburg, 1996; Seiwa, 2000; Parciak, 2002). Sin embargo también existen trabajos donde no se encontraron relación entre ambas variables, por ejemplo en Pinus sylvestris, Crisophyllum sp. nov., Convallaria majalis y Juglans ailanthifolia (Wrzesniewski, 1982; Eriksson, 1999; Green, 1999; Seiwa, 2000).

En el presente estudio se analizó la variación intraespecífica del tamańo de la semilla de Vismia glaziovii Ruhl. y su relación con la velocidad de germinación y el tamaño de las plántulas.

\section{Material y métodos}

Vismia glaziovii es una especie arbórea utilizada como recurso maderable en el Parque Nacional y Area Natural de Manejo Integrado Cotapata (PN ANMI Cotapata) en La Paz - Bolivia. El estudio se llevó a cabo en la Estación Biológica Tunquini (EBT) que se encuentra en el Bosque Húmedo Montañoso de Yungas a $1550 \mathrm{~m}$ de altitud, al sur oeste del PN ANMI Cotapata

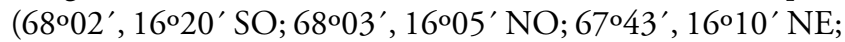
67047', 16²18'SE; Ribera, 1995; Moraes, 2001).

Estos bosques prosperan en condiciones mesohídricas y presentan una estacionalidad determinada por las precipitaciones (Ribera, 1995). En la EBT, el promedio anual de precipitación es $2900 \mathrm{~mm}$ y la temperatura promedio es aproximadamente 20 ${ }^{\circ} \mathrm{C}$ (EBT, 2001-2005 no pub.). El bosque es de mediana altura, con un estrato superior $(15-20 \mathrm{~m})$ denso y continuo, donde algunos géneros comunes son Juglans, Brunellia, Clusia, Cecropia, Psychotria, Miconia y Vismia, entre otros (Ribera, 1995). 
Tabla 1. Promedio del tamaño de semillas por fruto y por árbol de $V$. glaziovii. $\mathrm{N}=10$ semillas. El volumen es expresado como el volumen de las semillas $\left(\mathrm{mm}^{3}\right) \pm$ su desviación estandar.

\begin{tabular}{lccccc}
\hline $\begin{array}{l}\mathbf{N}^{\circ} \text { de } \\
\text { árbol }\end{array}$ & Fruto $\mathbf{1}$ & Fruto 2 & Fruto 3 & Fruto 4 & $\begin{array}{c}\text { Tamaño de las } \\
\text { semillas por } \\
\text { fruto por árbol }\end{array}$ \\
\hline 1 & $1,27 \pm 0,39$ & $1,44 \pm 0,30$ & $1,20 \pm 0,41$ & $1,30 \pm 0,35$ & $1,29 \pm 0,10$ \\
2 & $1,21 \pm 0,31$ & $1,13 \pm 0,17$ & $1,24 \pm 0,13$ & $1,30 \pm 0,20$ & $1,22 \pm 0,07$ \\
3 & $1,36 \pm 0,33$ & $1,40 \pm 0,30$ & $1,25 \pm 0,21$ & $1,61 \pm 0,33$ & $1,41 \pm 0,15$ \\
4 & $1,35 \pm 0,48$ & $1,14 \pm 0,23$ & $1,18 \pm 0,17$ & $1,09 \pm 0,23$ & $1,19 \pm 0,11$ \\
5 & $1,00 \pm 0,32$ & $0,93 \pm 0,21$ & $1,01 \pm 0,35$ & $1,08 \pm 0,16$ & $1,01 \pm 0,66$ \\
6 & $1,05 \pm 0,17$ & $1,01 \pm 0,28$ & $1,27 \pm 0,33$ & $1,09 \pm 0,23$ & $1,11 \pm 0,12$ \\
7 & $1,25 \pm 0,23$ & $1,12 \pm 0,19$ & $1,71 \pm 0,36$ & $1,06 \pm 0,19$ & $1,29 \pm 0,30$ \\
8 & $1,36 \pm 0,26$ & $1,16 \pm 0,25$ & $1,42 \pm 0,47$ & $1,36 \pm 0,09$ & $1,32 \pm 0,11$ \\
9 & $1,40 \pm 0,27$ & $1,27 \pm 0,32$ & $1,05 \pm 0,24$ & $1,14 \pm 0,25$ & $1,21 \pm 0,15$ \\
10 & $1,02 \pm 0,21$ & $0,96 \pm 0,29$ & $1,09 \pm 0,23$ & $1,17 \pm 0,17$ & $1,06 \pm 0,09$ \\
11 & $1,11 \pm 0,27$ & $1,16 \pm 0,17$ & $1,32 \pm 0,22$ & $1,17 \pm 0,19$ & $1,19 \pm 0,09$ \\
12 & $0,72 \pm 0,37$ & $1,71 \pm 0,31$ & $1,55 \pm 0,23$ & $1,37 \pm 0,26$ & $1,59 \pm 0,16$ \\
13 & $1,91 \pm 0,19$ & $1,50 \pm 0,33$ & $1,24 \pm 0,31$ & $1,85 \pm 0,39$ & $1,63 \pm 0,32$ \\
14 & $1,03 \pm 0,27$ & $1,27 \pm 0,24$ & $1,00 \pm 0,20$ & $1,06 \pm 0,21$ & $1,09 \pm 0,12$ \\
15 & $1,20 \pm 0,31$ & $2,27 \pm 0,29$ & $1,34 \pm 0,25$ & $1,24 \pm 0,15$ & $1,26 \pm 0,06$ \\
16 & $1,11 \pm 0,19$ & $1,14 \pm 0,27$ & $1,21 \pm 0,26$ & $1,38 \pm 0,25$ & $1,21 \pm 0,12$ \\
17 & $1,63 \pm 0,30$ & $1,74 \pm 0,34$ & $1,35 \pm 0,20$ & $1,41 \pm 0,20$ & $1,53 \pm 0,18$ \\
18 & $1,23 \pm 0,28$ & $1,31 \pm 0,21$ & $1,33 \pm 0,32$ & $1,18 \pm 0,37$ & $1,30 \pm 0,10$ \\
\hline
\end{tabular}

Vismia glaziovii es una especie arbórea heliófita, pionera y típica de bosque secundario (Guariguata y Ostertag, 2002). El fruto es una baya indehiscente, contiene resina anaranjada, está sostenido por el cáliz y coronado por los estilos pesistentes (Roca, 1993). En la EBT, la floración ocurre desde octubre a diciembre y la fructificación entre febrero y mayo (Arteaga, 2004). Los frutos tienen un volumen promedio de $1,27 \pm 0,3 \mathrm{~cm}^{3}$ y contienen, en promedio, 80,6 $\pm 26,9$ semillas $(C V=33 \%$; Arteaga, 2004). Estos frutos son consumidos principalmente por murciélagos (Aguirre et al., 2006).

A fines de marzo de 2002, de 18 árboles se colectaron aleatoriamente cuatro frutos maduros. De cada fruto se separaron diez semillas, obteniendo así un total de 720 semillas. Se midió el largo y diámetro de cada semilla con una precisión de $0,01 \mathrm{~mm}$. El 30 de marzo, todas las semillas fueron colocadas en cajas petri con algodón húmedo y con la tapa superior perforada para permitir la evaporación. Cada semilla dentro de cada caja fue numerada para su posterior seguimiento. Las cajas petri fueron ubicadas en un vivero al aire libre en la EBT. El vivero fue construido con malla milimétrica y techo transparente, para evitar la influecia directa de agua de lluvia. Las cajas fueron regadas cada 5 días y se revisaron diariamente para contar las semillas germinadas.

De las semillas germinadas, se transplantaron 250 plántulas a bolsas de polietileno $(250 \mathrm{ml})$ con tierra cernida, manteniendo la numeración de cada semilla para continuar con el seguimiento en el estadio de plántula. Se perforó cada bolsa cerca de la base para permitir la liberación de exceso de agua. Posteriormente, se colectaron aquellas plántulas que cumplieron 135 días después de la germinación $(\mathrm{N}=111)$. Esto debido a que, al haber trabajado en un vivero al aire libre, las condiciones ambientales influiran en el crecimiento de las plántulas, por esto se consideró la cohorte con mayor número de semillas germinadas un mismo día.

Se registró la longitud total de cada plántula, longitud del tallo y longitud de la raiz (Cuya y Lombardi, 1991). Posteriormente, las plántulas fueron secadas en un horno durante 48 horas a 70 ${ }^{\circ} \mathrm{C}$ (Hayashida-Oliver et al., 2001). Las muestras secas fueron pesadas (biomasa plántula, tallo, raiz y hojas, por separado) en una balanza analítica con una precisión de 0,00001 g, en la ciudad de La Paz - Bolivia.

Para el análisis de los datos, se calculó el volumen de cada semilla aproximandolo al volumen de un cilindro según la formula:

$$
\text { Volumen semilla }=\operatorname{largo} \pi^{*}(\text { diámetro/2) }
$$

La variación del volumen de las semillas entre árboles y entre frutos dentro de cada árbol fue analizada mediante el modelo de ANDEVA anidado (Sokal y Rohlf, 1998).

Considerando que el estudio busca encontrar relaciones de causa efecto entre el tamaño de la semilla y las variables dependientes de velocidad de germinación y tamaño de la plántula, se realizaron análisis mediante modelos de regresión lineal (Sokal y Rohlf, 1998; Gotelli y Ellison, 2004). Para esto, se consideraron el volumen de la semilla y las distintas variables de respuesta obtenidas en el experimento: días de germinación, medidas de longitud (plántula, tallo y raíz) y biomasa en peso seco (plántula, tallo, raíz y hojas). Las variables referidas al volumen de las semillas y el número de días de germinación fueron transformadas para que se ajusten a una distribución normal. La primera variable fue transformada utilizando $\log _{10}$ y para la segunda variable se utilizó su raiz cuadrada (Gotelli y Ellison,2004). Se eligió un $\alpha$ de 0,05 para que las diferencias fueran consideradas estadísticamente significativas.

\section{Resultados y discusión}

Se determinó que la variación del tamaño de las semillas entre árboles no es significativa $\left(\mathrm{F}_{17,648}=0,68 ; \mathrm{p}=0,825\right.$; Tabla 1$)$. Por el contrario, existe diferencias significativas al comprar el tamaño de las semillas entre frutos dentro de cada árbol $\left(\mathrm{F}_{54,648}=1554,01\right.$; $\mathrm{p}<0,05$; Tabla 1). La mayor variabilidad se registró entre las semillas dentro de cada fruto, aportando una varianza de 0,21 , mientras que el aporte de la varianza de la diferencia entre frutos es 0,048 (MS frutos - MS semillas)/10; CV =16,92\%).

De las 720 semillas que se pusieron a germinar, $591(82,1 \%)$ germinaron en un lapzo de 79 días. Las semillas empezaron a germinar el sexto día, a partir del cuál se dio un incremento paulatino de semillas germinadas. 


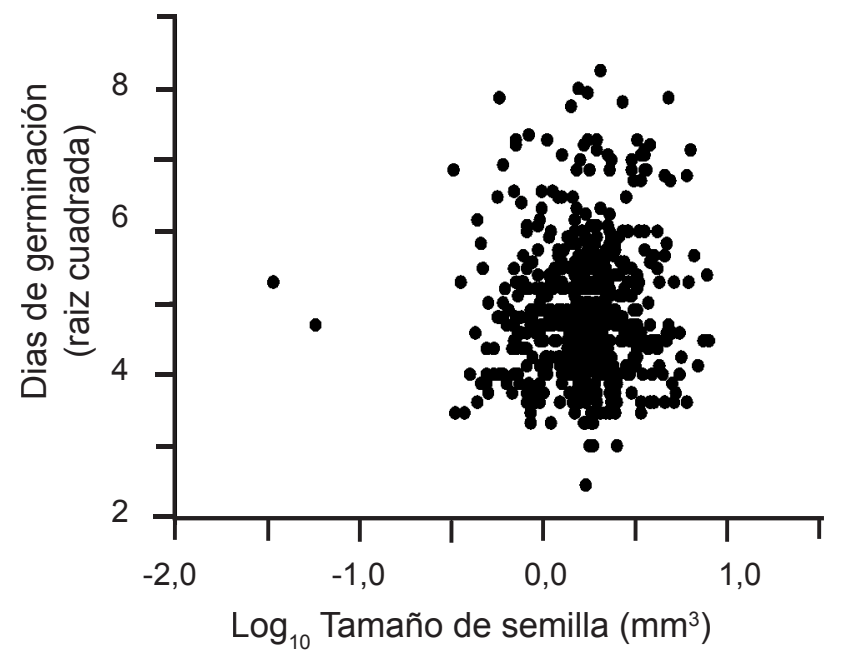

Figura 1. Relación entre el tamaño de la semilla y la velocidad de germinación en Vismia glaziovii $\left(r^{2}=0,019 ; p=0,150 ; N\right.$ =591).

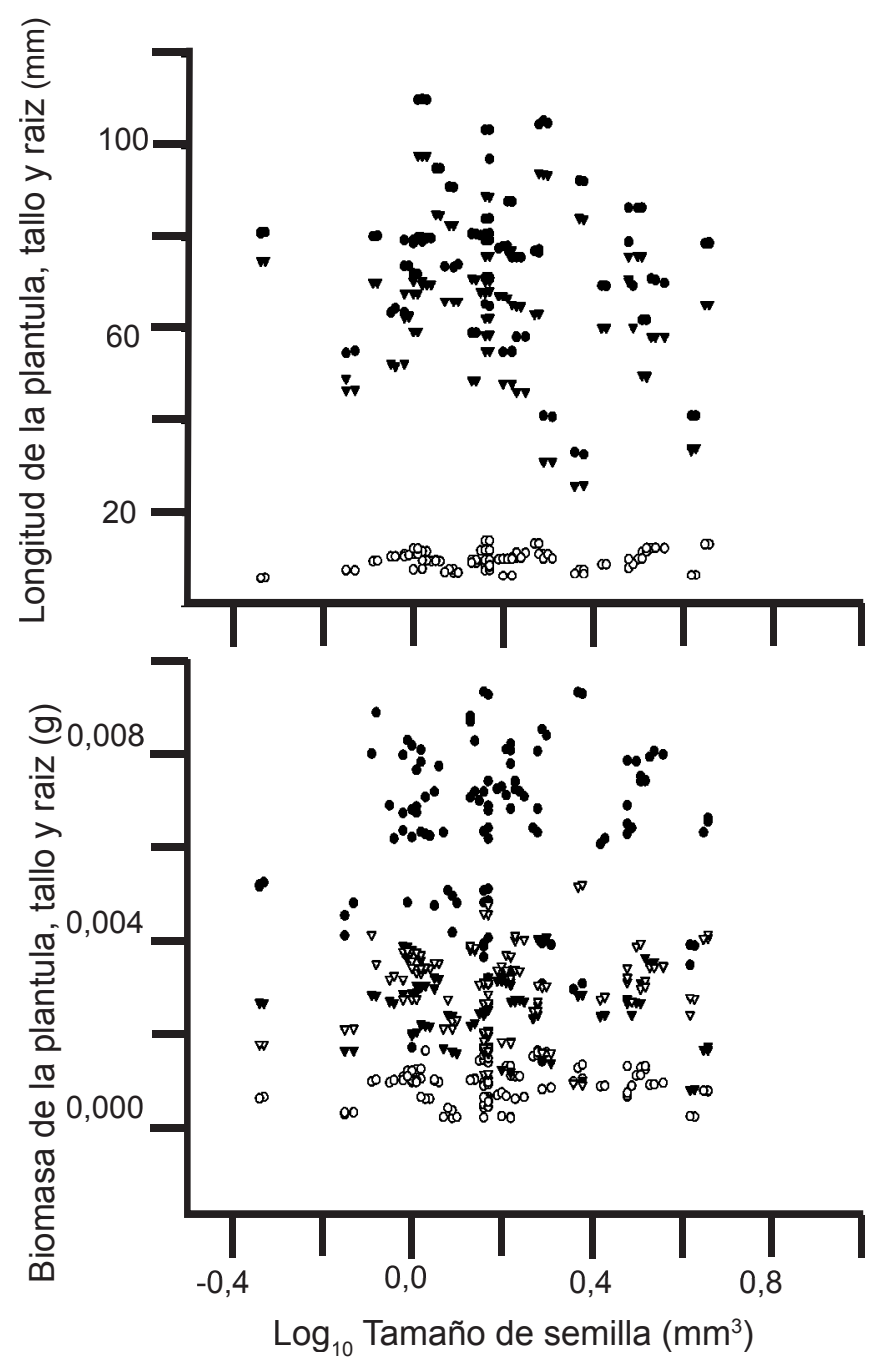

Figura 2. Relación entre el volúmen de las semillas y el tamaño de las plántulas en Vismia glaziovii $(\mathrm{N}=111)$ : a) longitud y b) peso. Circulos cerrados $=$ plántula $\left(\right.$ longitud $r^{2}=0,04 ; p=0,32$; peso $r^{2}=0,01 ; p=0,47$ ); triángulos cerrados = raiz (longitud $r^{2}=0,06 ; p=0,13$; peso $\left.r^{2}=0,02 ; p=0,16\right)$; circulos abiertos $=$ tallo (longitud $r^{2}=0,04 ; p=0,48 ;$ peso $r^{2}=0,01 ; p=0,46$ ); triángulos abiertos $=$ hojas $\left(\right.$ peso $\left.r^{2}=0,02 ; p=0,14\right)$.
No se encontró relación entre el $\log _{10}$ del tamaño de la semilla y la velocidad de germinación $\left(\mathrm{r}^{2}=0,019 ; \mathrm{p}=0,150\right.$; Fig. 1), ni con las distintas medidas referidas al tamaño de las plántulas (longitud y biomasa; Fig. 2a y b).

Determinar que el tamaño de las semillas de $V$. glaziovii no varía significativamente entre árboles y que la diferencia es significativa al comparar las semillas entre frutos de un mismo árbol, concuerda con lo propuesto por Michaels et al. (1988) y Vaughton y Ramsey (1998), quienes dicen que es más probable encontrar diferencia en el tamańo de la semilla dentro de una planta que entre plantas, indicando que la variación se debería más a efectos medioambientales durante el desarrollo que a diferencias genéticas entre árboles parentales. En el caso de $V$. glaziovii, al ser su fruto una baya, es probable que además de las condiciones ambientales que rodean a cada individuo exista un efecto de la posición de las semillas dentro del fruto, ya que esto puede afectar el tamaño y la morfología de las semillas (Gutterman, 1992; Eriksson, 1999).

Si bien existe variación del tamaño de la semilla dentro de los frutos, dicha varibilidad parece no influir sobre la velocidad de germinación ni con el tamaño de la plántula. Debido a que V. glaziovii presenta semillas muy pequeńas $\left(\mathrm{X}=1,27 \mathrm{~mm}^{3}\right)$, es probable que la variación existente en el tamaño de las semillas no exprese una diferencia significativa en la cantidad de recurso almacenado que pueda influir en la velocidad de germinación y el tamańo de las plántulas. Por lo tanto, es probable que no exista dependencia del tamańo de la semilla y las concentraciones de proteínas y carbohidratos, factores que influyen en la germinación (Tripathi y Khan, 1990).

Respecto a la variación encontrada en el tiempo de germinación $(24,7 \pm 10,2$ días), es probable que ésta se deba tambien a características de ubicación y maduración de las semillas dentro de los frutos, ya que la posición de la infrutescencia en la planta o la posición de las semillas en el fruto puede influir sobre la germinabilidad de las semillas (Grey y Thomas, 1982; Gutterman, 1992). Es así que, en algunas especies, la influencia del grado de desarrollo del embrión sobre la velocidad de germinación y tamaño de la plántula puede ser mayor que el efecto del tamaño de las semillas (Wrzesniewski, 1982).

\section{Agradecimientos}

Agradezco a R. Bustamante, L. Pacheco, A. Roldán, B. Mostacedo y los revisores anónimos por la revisión y sugerencias hechas al presente documento. De igual manera, agradezco a I. Moya, F. Montaño, N. Burgoa, E. Perez, F. Paredes, R. Ticona y J. Torres por la colaboración en el trabajo de campo y vivero en la EBT. Al Laboratorio de Suelos del Instituto de Ecología por permitirme utilizar las balanzas analíticas en La Paz. El trabajo lo realicé con el apoyo de la Fundación John D. and Catherine T. MacArthur a la Estación Biológica Tunquini. La EBT agradece a Alejandra Roldán por escribir el proyecto que dio lugar a la realización de este trabajo.

\section{Literatura citada}

Aguirre, G., L.L. Arteaga y M.I. Moya. 2006. Proporción y velocidad de germinación de semillas de Vismia glaziovii (Guttiferae) obtenidas de las heces de murciélagos frugívoros. Revista Boliviana de Ecología y Conservación Ambiental 19: 101-106. 
Arteaga, L.L. 2004. Ecología de poblaciones de especies forestales escogidas. Informe Técnico. La Paz: Estación Biológica Tunquini - Instituto de Ecología.

Bockus, W.W. y J.P. Shroyer. 1996. Effect of seed size on seedling vigor and forage production of winter wheat. Canadian Journal of Plant Science 76: 101-105.

Cuya, O. y I. Lombardi. 1991. Influencia del tamaño de semilla en la germinación y crecimiento de plántulas de Schinus molle. Revista Forestal del Peru 18(2): 17-27.

Dalling, J.W. 2002. Ecología de semillas. En: M. Guariguata y G. Catan, eds. Ecología y Conservación de Bosques Neotropicales. Libro Universitario Regional, Cartago, Costa Rica. Pp. 345-375.

Dolan, R.W. 1984. The effect of seed size and maternal source on individual size in a population of Ludwigia leptocarpa (Onagraceae). American Journal of Botany 71: 1302-1307.

Eriksson, O. 1999. Seed size variation and its effect on germination and seedling performance in the clonal herb Convallaria majalis. Acta Oecologica 20(1): 61-66.

González, E. 1993. Effect of seed size on germination and seedling vigor of Virola koschny Warb. Forest Ecology and Management 57: 275-281.

Gotelli, N.J. y A.M. Ellison. 2004. A primer of ecological statistics. Sinauer Associates, USA. 510 p.

Green, P.T. 1999. Seed germination in Chrysophyllum sp nov., a largeseeded rainforest species in north Queensland: effects of seed size, litter depth and seed position. Australian Journal of Ecology 24: 608-613.

Grey, D. y T.H. Thomas. 1982. Seed germination and seedling emergence as influenced by the position of development of the seed on the parent plant. En: A. Khan, ed. The physiology and Biochemistry of seed Development, Dormancy and Germination. Elsevier, Nueva York, Pp. 81-110.

Guariguata, M.R. y R. Ostertag. 2002. Sucesión secundaria. En: M. Guariguata y G. Catan, eds. Ecología y Conservación de Bosques Neotropicales. Libro Universitario Regional, Cartago, Costa Rica. Pp. 451-623.

Gutterman, Y. 1992. Maternal effects on seeds during development. En: M. Fenner, ed. Seeds: The Ecology of Regeneration in Plant Communities. CAB International, Redwood Press, Melksham. Pp. 27-59.

Hartmann, H. y D.E. Kester. 1982. Propagación de plantas. México: CECSA.

Hayashida-Oliver, Y., R. Boot y L. Poorter. 2001. Influencia de disponibilidad de agua y luz en el crecimiento y la morfología de plantines de Swietenia macrophylla, Cedrela odorata y Bertholletia excelsa. Ecología en Bolivia 35: 51-60.

Howe, H.F. y W. Richter. 1982. Effects of seed size on seedling size in Virola surinamensis: a within and between tree analysis. Oecologia 53: 347-351.

Landford, G.P. y R. J. Baker. 1986. Effects of genotype and seed size on speed of emergence and seedling vigor in nine spring wheat cultivars. Crop Science 26: 341-346.
Leishman, M.R., I.J. Wright, A.T. Moles y M. Westoby. 2000. The evolutionary ecology of seed size. En: M. Fenner, ed. Seeds: The Ecology of Regeneration in Plant Comunities, 2nd edition. CAB International, Redwood Press, Melksham. Pp. 31-57.

Michaels, H.J., B. Benner, A.P. Hatgerink, T.D. Lee, S. Rice, M. F. Willson y R.I. Bertin. 1988. Seed size variation: magnitude, distribution, and ecological correlates. Evolutionary Ecology 2: 157-166.

Moegenburg, S.M. 1996. Sabal palmetto seed size - causes of variation, chaices of predators, and consequences for seedlings. Oecologia 106: 539-543.

Moraes, M. 2001. La Estación Biológica de Tunquini: Un sitio favorable para entrenamiento e investigación biológica y ecológica de los bosques montanos de Bolivia. Ecología en Bolivia 36: 1-2.

Parciak, W. 2002. Seed size, number, and habitat of a fleshy-fruited plant: consequences for seedling establishment. Ecology 83(3): 794-808.

Ribera, M.O. 1995. Aspectos ecológicos del uso de la tierra y conservación en el Parque Nacional y Area de Manejo Integrado Cotapata. En: C. de Morales, ed. Caminos de Cotapata. IE, Fund-Eco, Fonama - EIA, La Paz. Pp. 1-46.

Roca, Y. 1993. Guttiferae. En: T. Killeen, E. Garcia y S. Beck, eds. Guía de árboles de Bolivia. La Paz: Herbario Nacional de Bolivia - Missouri Botanical Garden. Pp. 337-351.

Seiwa, K. 2000. Effects of seed size and emergence time on tree seedling estblishment: importance of developmental constraints. Oecologia 123: 208-215.

Snow, D.W. 1971. Evolutionary aspects of fruit-eating by birds. Ibis 113: 194-202.

Sokal, R. y F. Rohlf. 1998. Biometry. Nueva York: Freeman \& Company.

Tripathi, R.S. y M.L. Khan. 1990. Effects of seed weight and microsite characteristics on germination and seedling fitness in two species of Quercus in a subtropical wet hill forest. Oikos 57: 289-296.

Vaughton, G. y M. Ramsey. 1998. Sources and consequences of seed mass variation in Banksia marginata (Proteaceae). Journal of Ecology 86: 563-573.

Winn, A.A. 1988. Ecological and evolutionary consequences of seed size in Prunella vulgaris. Ecology 69: 1537-1544.

Winn, A.A. 1991. Proximate and ultimate sources of within-individual variation in seed mass in Prunella vulgaris (Lamiaceae). American Journal of Botanic 78: 838-844.

Wrzesniewski, W. 1982. Physiology of Scots pine seedlings grown from seed of different weight. I. Differentiation of seed characteristics. Acta Physiological Plant 4: 31-42.

Wulff, R.D. 1986. Seed size variation in Desmodium paniculatum: Effects on seedling growth and physiological performance. Journal of Ecology 74: 99-114.

Zhang, J. y M.A. Maun. 1991. Establishment and growth of Panicum virgatum L. seedlings on a Lake Erie sand dune. Bulletin of the Torrey Botanical Club 118: 141-153. 